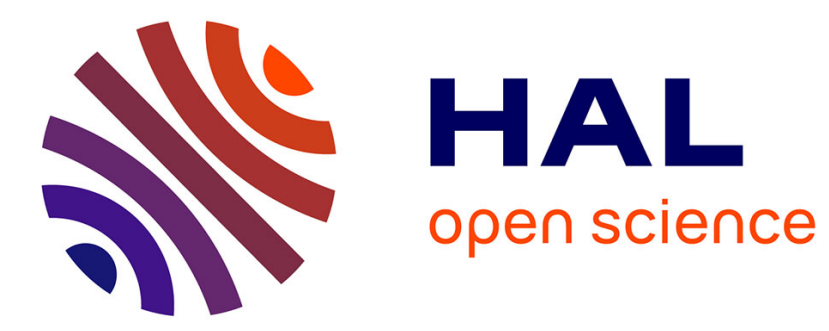

\title{
An Alternative Formulation for the Empirical Mode Decomposition
}

\author{
Thomas Oberlin, Sylvain Meignen, Valérie Perrier
}

\section{To cite this version:}

Thomas Oberlin, Sylvain Meignen, Valérie Perrier. An Alternative Formulation for the Empirical Mode Decomposition. IEEE Transactions on Signal Processing, 2012, 60 (5), pp.2236-2246. 10.1109/TSP.2012.2187202 . hal-00553107

\section{HAL Id: hal-00553107 https://hal.science/hal-00553107}

Submitted on 6 Jan 2011

HAL is a multi-disciplinary open access archive for the deposit and dissemination of scientific research documents, whether they are published or not. The documents may come from teaching and research institutions in France or abroad, or from public or private research centers.
L'archive ouverte pluridisciplinaire HAL, est destinée au dépôt et à la diffusion de documents scientifiques de niveau recherche, publiés ou non, émanant des établissements d'enseignement et de recherche français ou étrangers, des laboratoires publics ou privés. 


\title{
An Alternative Formulation for the Empirical Mode Decomposition
}

\author{
Thomas Oberlin \\ LJK Laboratory, University of Grenoble, France \\ Tel:0033-4-76-51-45-61 \\ E-mail: thomas.oberlin@imag.fr \\ Sylvain Meignen \\ LJK Laboratory, University of Grenoble, France \\ Tel:0033-4-76-51-43-95 \\ FAX:0033-4-76-63-12-63 \\ E-mail: sylvain.meignen@imag.fr \\ Valérie Perrier \\ LJK Laboratory, University of Grenoble, France \\ Tel:0033-4-76-51-45-51
}

FAX:0033-4-76-63-12-63

E-mail: valerie.perrier@imag.fr

\begin{abstract}
Empirical Mode Decomposition (EMD) is a relatively new method for adaptive multiscale signal representation. As it allows to adaptively analyze nonlinear and non-stationary signals, it is widely used in signal processing. Yet, as the standard EMD method lacks a solid mathematical background, many alternative constructions have been proposed to define similar decompositions in a more comprehensive way. This paper is in line with this idea, as it defines a new decomposition that lies on direct constrained optimization. We show that this new approach gives satisfactory results for narrow-band signals and preserves the essential characteristics of the original EMD.
\end{abstract}

Keywords- Empirical Mode Decomposition, Constrained B-splines, Adaptive signal decomposition, Adaptive multiscale analysis

EDICS Category: DSP-TFSR 


\section{INTRODUCTION}

The Empirical Mode Decomposition (EMD), introduced by Huang et al. [1], is a now well-known data-driven signal processing method. Due to its remarkable efficiency, applications of the EMD cover many signal processing areas [2], [3]. However, the method is basically an iterative algorithm, called the sifting process (SP), lacking clear mathematical foundations. Although the SP works quite well in many practical cases, it depends on ad-hoc parameters. These last years, some authors have proposed to replace the SP by more formal tools, like partial differential equations [4], [5], or by optimization methods [6], [7]. Some of these alternative approaches sometimes give interesting results, but usually do not reproduce the main characteristics of the original EMD. Conversely, some other approaches build improved versions of the SP, by changing the definition of the signal local mean [8], or by refining the interpolation technique [9].

This paper introduces a new formulation for the EMD that takes advantage of recent advances on the definition of the signal local mean and that does not lie on the SP. The basic idea of the method is to replace the SP by a constrained optimization procedure putting a particular emphasis on the initialization issue. A comparison with other existing methods is carried out in terms of separability of the modes and of orthogonality index.

\section{EMPIRICAL MOde DeCOMPOSITION}

\section{A. Presentation}

Any signal $s$ can be split into an oscillating part $h$, and a less oscillating local mean $m$. However, such a decomposition may not have any physical meaning. Following this idea, the EMD aims at adaptively decomposing a signal $s$ into a finite sum of modes $h_{1}, \ldots, h_{N}$ and a residue $r$ as follows:

$$
s=\sum_{k=1}^{N} h_{k}+r .
$$

The modes $h_{k}$, called Intrinsic Mode Functions (IMF's), are less oscillating with increasing $k$, whereas the residue, representing the mean trend of the signal, is monotonic.

In the original method, the IMF's are defined as "symmetrical oscillating functions": an IMF is a function with positive maxima and negative minima, whose upper envelope (built by interpolation of the maxima) is symmetric with respect to its lower envelope. The Hilbert transform of the IMF is then used to define the instantaneous frequency of the signal. The proposed definition of the IMF's is sound since these are AM/FM signals with small amplitude and frequency modulation, leading to meaningful Hilbert transform due to the Bedrosian identity [10]. 


\section{B. The Sifting Process}

To extract each IMF, the EMD uses an iterative procedure called the sifting process (SP), which consists in recursively subtracting the "local mean" of the signal. In that context, it is defined as the average of the upper and of the lower signal envelopes. More precisely, given a signal $s$, the SP extracts the first IMF $h$ by putting $h:=s$, and by recursively computing

$$
h:=\phi(h)=h-\frac{E_{\max }(h)+E_{\min }(h)}{2} .
$$

where $E_{\min }(h)$ (resp. $E_{\max }(h)$ ) is the cubic spline interpolant at the minima (resp. maxima) of $h$. It is clear that the convergence of the process would imply that $h_{\infty}$ has symmetrical envelopes. However, in a cubic spline interpolation framework, the symmetry of the envelopes requires that these are cubic polynomials on the whole time span [11]. Consequently, the symmetry criterion is much too restrictive. Therefore, the standard EMD only iterates $\phi K$ times, $K$ being imposed by an ad-hoc stopping criterion. The stopping criterion can be of Cauchy-type, like in the original definition [1], or it can be related to the symmetry of the envelopes [12]. The main drawback of the method is that, given one of these stopping criteria and a signal, there is no theoretical proof that the SP should stop in a finite number of steps $K$.

\section{Properties of the EMD}

We saw that the EMD is a nonlinear adaptive decomposition method. The two main properties of the decomposition are as follows:

- The EMD has a multiscale nature: the local scale parameter (linked to the instantaneous frequency) decreases through the sequence $\left(h_{1}, h_{2}, \ldots\right)$. It is even shown in [13] that the EMD behaves on average like a dyadic filter bank.

- The EMD is a quasi-orthogonal decomposition [1], but no proof of this result is available.

Although these properties are mostly empirical, they make the EMD belonging to the class of multiscale, orthogonal and adaptive decompositions. In this regard, and from a practical point of view, any change in the original formulation should preserve these characteristics.

\section{OPTIMIZATION-BASED EMD FOR NARROW-BAND SIGNALS}

In this section, we propose a new approach for the EMD based on constrained optimization that is mathematically sound and that preserves most of the interesting properties of the original one. 


\section{A. Description}

The method consists in finding a spline $m$ of order $k$, which is close to the local mean of the signal $s$. It will be searched in the space $\Pi_{\tau}^{k}$ : the B-splines of order $k$ on a subdivision $\tau$.

We will first build the knot sequence $\tau$ and an approximation $m_{0}$ of the local mean, and then design a set of constraints $C$ and a functional $J$ which serve to compute $m$ and the first IMF $h=s-m$. The optimization problem may formally be written as:

$$
\hat{m}=\left\{\begin{array}{c}
\operatorname{argmin} J(m) \\
m \in \Pi_{\tau}^{k} \cap C .
\end{array},\right.
$$

In the following paragraphs, we describe the different steps of the method.

1) Computation of $m_{0}$ and the B-splines space: Let us consider $\left(\hat{\theta}_{i}\right)_{i=1 \ldots L}$ an estimate sequence of the location of the extrema of the high frequency mode: they are most commonly the extrema of the signal, but can be of other types, as it will be explained later. To build an approximation $m_{0}$ of the local mean of the signal, we first follow the approach of [8], which defines the points $\left(\bar{s}_{i}, \bar{t}_{i}\right)_{i=1 \ldots L-1}$ by:

$$
\begin{aligned}
\bar{s}_{i} & =\frac{1}{\hat{\theta}_{i+1}-\hat{\theta}_{i}} \int_{\hat{\theta}_{i}}^{\hat{\theta}_{i+1}} s(t) d t \\
\bar{t}_{i} & =\frac{\int_{\hat{\theta}_{i}}^{\hat{\theta}_{i+1}} t\left|s(t)-\bar{s}_{i}\right|^{2} d t}{\int_{\hat{\theta}_{i}}^{\hat{\theta}_{i+1}}\left|s(t)-\bar{s}_{i}\right|^{2} d t} .
\end{aligned}
$$

In [8], this computation of the mean envelope replaced the original one in the sifting process. The numerical results showed better modes separation and less sensitivity to noise. Let us now detail our construction of $m_{0}$ which is based on B-spline interpolation.

In order to define $m_{0}$ on the whole time span, supposed to be $[0,1]$, we symmetrize $\left(\bar{s}_{1}, \bar{t}_{1}\right)$ with respect to $\hat{\theta}_{1}$, to get $\left(\bar{s}_{0}, \overline{t_{0}}\right)$, and we similarly define the last point $\left(\bar{s}_{L}, \overline{t_{L}}\right)$. We then want $m_{0}$ to be a B-spline interpolating these $L+1$ points. We compute after the $\bar{t}_{i}$ a knot sequence $\left(\tau_{i}\right)_{i=0 \ldots L+k}$ using the classical definition [14]:

- we use multiple knots at the boundaries: $\tau_{0}=\ldots=\tau_{k-1}=\bar{t}_{0}$ and $\tau_{L+1}=\ldots=\tau_{L+k}=\bar{t}_{L}$,

- and the following interior single knots:

$$
\forall i \in k \ldots L, \tau_{i}=\frac{1}{k-1} \sum_{j=i+1-k}^{i-1} \bar{t}_{j} .
$$

Note that (4) implies that

$$
\forall i \in 0 \ldots L, N_{i, \tau}^{k}\left(\bar{t}_{i}\right)>0
$$


where $N_{j, \tau}^{k}$ is the $j^{t h}$ B-spline of order $k$ on the subdivision $\tau$, with support $\left[\tau_{j}, \tau_{j+k}\right]$. With these condition, the Schoenberg-Whitney theorem shows that there is a unique interpolation spline of order $k$ at the locations $\bar{t}_{i}$. Note also that the subdivision is as fine as the local scale of the high frequency mode, so that the error made by the spline interpolation of the low frequency part is small.

With our formalism, the function $m$ takes the following form:

$$
m=\sum_{i=0}^{L} M_{i} N_{i, \tau}^{k},
$$

so that the optimization problem amounts to finding the weights $M_{i}$.

2) The constraints: In the original EMD algorithm, the IMF $h$ is defined by the symmetry of its upper and lower envelopes and by the zero-crossing property: all the maxima are positive and all the minima are negative.

Let us assume that the extrema of $h$ are located at $\left(x_{i}\right)_{i=1 \ldots L}$. At each point $x_{i}$, we define a linear inequality constraint associated to the symmetry of the upper and lower envelopes of $h$. Indeed, let us define $\lambda_{i}$ the point with abscissa $x_{i}$ on the linear envelope passing through $\left(x_{i-1}, h\left(x_{i-1}\right)\right)$ and $\left(x_{i+1}, h\left(x_{i+1}\right)\right)$ (see Fig. 1):

$$
\lambda_{i}=\frac{h\left(x_{i+1}\right)-h\left(x_{i-1}\right)}{x_{i+1}-x_{i-1}}\left(x_{i}-x_{i-1}\right)+h\left(x_{i-1}\right) .
$$

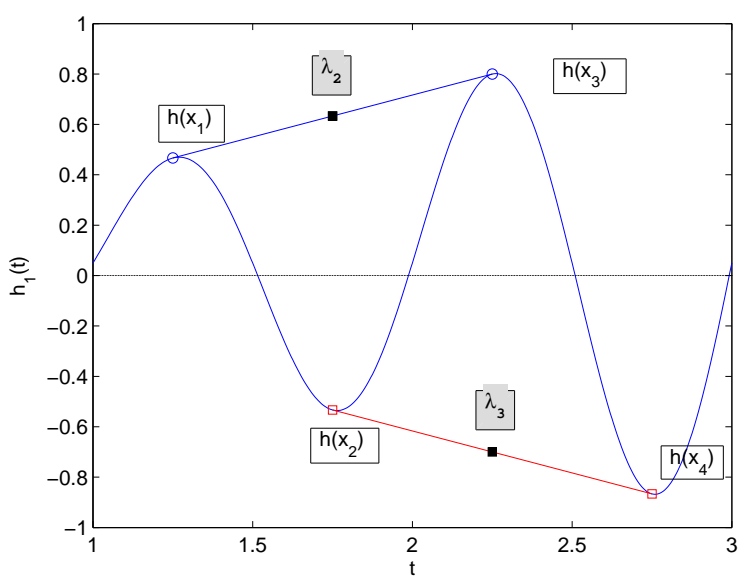

Fig. 1. Definition of $\lambda_{i}$

Of course this definition is valid only for $i=2, \cdots, L-1$. We also define $\lambda_{1}=h\left(x_{2}\right)$ and $\lambda_{L}=$ $h\left(x_{L-1}\right)$, which amounts to symmetrizing $h$ with respect to its first and last extrema. Note that $\lambda_{i}$ depends linearly on $h$, and also on $m$, so that it will be denoted by $\lambda_{i}(m)$. 
To take into account the symmetry of the envelopes of $h$, we will impose

$$
\left|h\left(x_{i}\right)+\lambda_{i}(m)\right| \leq \varepsilon_{i}
$$

As the threshold $\varepsilon_{i}$ shall be dependent on the local amplitude of $h$, it is natural to choose

$$
\varepsilon_{i}=\alpha\left|h\left(x_{i}\right)-\lambda_{i}(m)\right|
$$

where $\alpha$ is a global parameter. This relation is very similar to that used in the stopping criterion proposed in [12]: the aim was, in that paper, to iterate the SP until the condition

$$
\sigma(h)(t)=\frac{\left|E_{\max }(h)(t)+E_{\min }(h)(t)\right|}{\left|E_{\max }(h)(t)-E_{\min }(h)(t)\right|} \leq \alpha
$$

was satisfied for almost all $t$ and where $E_{\max }(h)$ and $E_{\min }(h)$ are defined at the beginning of section II.B. Our approach is novel in the following two aspects: first, the constraints have to be verified for all $x_{i}$ 's but not for all $t$ and second, the computation of $h$ will not be done through an iterative procedure.

Thus defined, the constraints imposed on $h$ are not linear since, on the one hand, the locations $\left(x_{i}\right)_{i=1, \cdots, L}$ of the extrema of $h$ are a priori unknown and, on the other hand, $\varepsilon_{i}$ depends on $h$. To cope up with these difficulties, we assume that $h_{0}=s-m_{0}$ is close enough to $h$ so that we can use some information on the former approximation to make the constraints linear. Moreover, we replace $x_{i}$ by the estimates $\hat{\theta}_{i}$. More precisely, the optimization problem will be the following:

$$
\hat{m}=\left\{\begin{array}{l}
\operatorname{argmin} J(m) \\
m \in \Pi_{\tau}^{k} \\
\forall i \in 1 \ldots L, \frac{\left|\lambda_{i}(m)+(s-m)\left(\hat{\theta}_{i}\right)\right|}{\left|\lambda_{i}\left(m_{0}\right)-\left(s-m_{0}\right)\left(x_{i}^{0}\right)\right|} \leq \alpha,
\end{array}\right.
$$

where $\left(x_{i}^{0}\right)_{i=1 \cdots L}$ are the extrema of $s-m_{0}$. In that context, it is crucial that $m_{0}$ be a correct estimation of the mean envelope of $s$.

Now, let us prove that the set $\mathrm{C}$ is non-empty. We will also show that there exists a spline $\tilde{m}$ of $\Pi_{\tau}^{k}$, with coefficients $\tilde{M}_{j}$, which satisfies $\lambda_{i}(\tilde{m})+(s-\tilde{m})\left(\hat{\theta}_{i}\right)=0, i=1 \ldots L$. Let us introduce some notations.

- $h(\hat{\theta})$ is the vector $\left(h\left(\hat{\theta}_{1}\right), \ldots, h\left(\hat{\theta}_{L}\right)\right)^{T}$.

- $\Lambda$ is the matrix building the $\lambda_{j}$ : we have $(\Lambda h(\hat{\theta}))_{j}=\lambda_{j}$.

- $P$ is the collocation matrix defined by $P_{i j}=N_{j, \tau}^{k}\left(\hat{\theta}_{i}\right)$. We have the relation $(P \tilde{M})_{j}=\tilde{m}\left(\hat{\theta}_{j}\right)$. We also have $\lambda_{i}(\tilde{m})=\Lambda(s(\hat{\theta})-P \tilde{M})$.

- $I_{L}$ is the identity matrix in $\mathbb{R}^{L \times L}$. 
Now, we see that $\tilde{m}$ exists if $\left(I_{L}+\Lambda\right)(s(\hat{\theta})-P \tilde{M})=0$. A sufficient condition on $\tilde{M}$ is $P \tilde{M}=s(\hat{\theta})$, which rewrites

$$
\forall i \in 1 \ldots L, s\left(\hat{\theta}_{i}\right)=\tilde{m}\left(\hat{\theta}_{i}\right)
$$

In section III-A1, we constrained the knot sequence $\tau$ to satisfy:

$$
\forall i \in 0 \ldots L, N_{i, \tau}^{k}\left(\bar{t}_{i}\right)>0
$$

This relation implies:

$$
\forall i \in 0 \ldots L, \tau_{i} \leq \bar{t}_{i} \leq \tau_{i+k},
$$

In addition, we have by definition of the $\bar{t}_{i}(3)$ that

$$
\forall i \in 1 \ldots L, \bar{t}_{i-1}<\hat{\theta}_{i}<\bar{t}_{i} .
$$

It follows that for $i \in 1 \ldots L$, either $N_{i-1, \tau}^{k}\left(\hat{\theta}_{i}\right)>0$ or $N_{i, \tau}^{k}\left(\hat{\theta}_{i}\right)>0$. By Schoenberg-Whitney theorem, there exists at least one spline of $\Pi_{\tau}^{k}$ (in fact, many) interpolating at the $\hat{\theta}_{i}$ and also satisfying (9).

In the constraints built here, the envelopes are computed by linear interpolation, whereas in the original formulation, smooth envelopes like cubic splines are used. We could wonder, whether to use cubic splines would improve the results. In this regard, let us first note that, in the original EMD, the mean envelope is iteratively subtracted to compute the IMF, so that smooth mean envelopes are needed. As we will see in section IV-B, our method does not need smooth envelopes, as it only imposes the symmetry at some locations. We thus expect that the error due to linear interpolation is small compared to the parameter $\alpha$. In practice, to use cubic spline envelopes improves the results so little, that one shall keep the linear definition.

3) The functional $J$ : In the original EMD formulation, the SP had two distinct effects: it constrained the modes to have approximative symmetrical envelopes while it had a regularization effect on the mean envelope $m$.

The constraints built in section III-A2 deal with the first aspect. The functional will involve the second one. It seems natural to choose the simple following term:

$$
J(m)=\left\|m^{\prime \prime}\right\|^{2},
$$

where $\|$.$\| is the classical L^{2}$ norm.

Among all possible local means designed by the constraints, we will choose the smoothest one. Let us notice that this functional is quadratic, leading to a unique and easily computable solution: if we denote 
by $\left(M_{j}\right)$ the coefficients of $m$, the functional rewrites as

$$
J(M)=M^{T} H M,
$$

with $H$ being the weight matrix defined by

$$
H_{i j}=\int_{0}^{1}\left(N_{i, \tau}^{k}\right)^{\prime \prime}(t)\left(N_{j, \tau}^{k}\right)^{\prime \prime}(t) d t .
$$

4) Estimation of the extrema $\hat{\theta}_{i}$ : First note that the constraints $C$ are meaningful only when the number of the extrema estimate $\hat{\theta}$ equals that of the HF component. Then, an accurate estimate $\hat{\theta}$ is all the more important since we use the approximation $m_{0}$ (depending on $\hat{\theta}$ ) in the optimization procedure (see formula (3)).

Note that, in the original formulation, the estimate $\hat{\theta}$ is set to the extrema of the signal. The role of the sifting process is then to iteratively move these points towards the locations of the extrema of the IMF. As in our optimization problem there is no iterative procedure, our concern is to detect with a very good accuracy all the extrema of the HF mode.

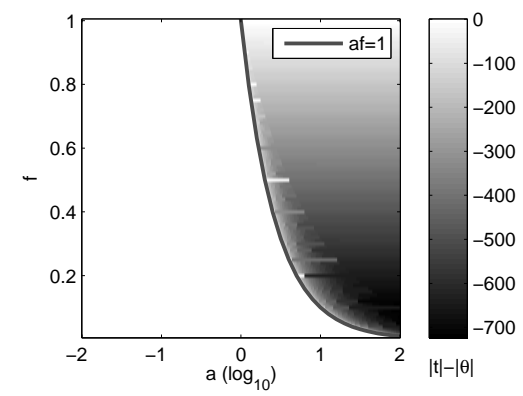

(A)

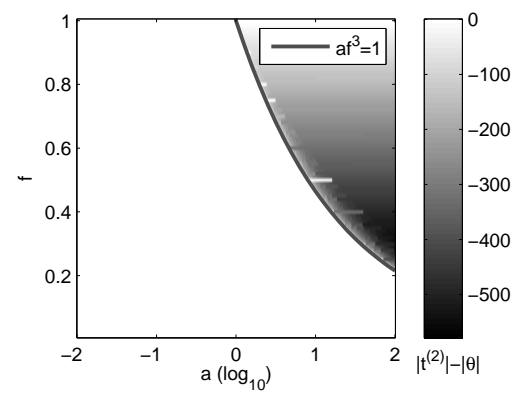

(B)

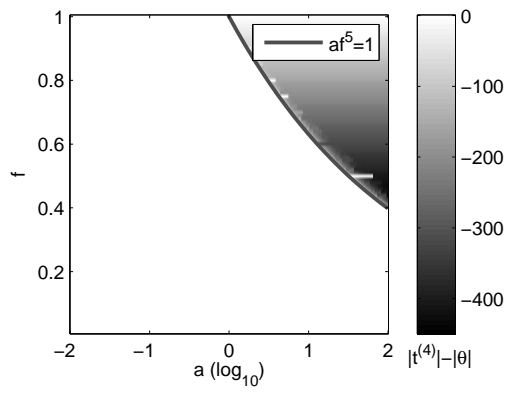

(C)

Fig. 2. (A): Difference $|t|-|\theta|$ between the number of extrema of the signal $s$ and that of the HF mode $s_{1}$, for different values of $a$ and $f$. The number of extrema of the HF mode is well determined by $|t|$ when $a f<1$ (white area). (B): the same computation using $t^{(2)}$ instead of $t$ : draw of $\left|t^{(2)}\right|-|\theta|$. The number of extrema of the HF component is well determined when $a f^{3}<1$. (C): the same computation using $t^{(4)}$ instead of $t$ : draw of $\left|t^{(4)}\right|-|\theta|$. The number of extrema of the HF component is well determined when $a f^{5}<1$.

As remarked in [9], the location of the extrema of even order derivatives of $s$ are more likely to provide a better approximation of the location of the extrema of $h$ than that of the original signal. In [9], the case of the estimation of the location of the extrema of $h$ by that of $s^{(2)}$ is investigated when $s$ is as follows:

$$
s(t)=\sum_{i=1}^{P} a_{i} \cos \left(2 f_{i} \pi t\right),
$$


where $f_{i}$ is assumed to be decreasing with $i$. It is proved there, using a maximal deviation argument that the locations of the extrema of $s_{1}(t)=a_{1} \cos \left(2 f_{1} \pi t\right)$ are better estimated by the location of the extrema of $s^{(2)}$ than by that of $s$. However, from a practical point of view, the maximal deviation may not be enough informative. To be convinced of that, let us consider the following simple two-tones signal:

$$
s(t)=\cos (2 \pi t)+a \cos (2 \pi f t) \quad 0<f<1 a \in \mathbb{R}_{+} .
$$

If we refer to Proposition 1 of [15], when af $<1$ the extrema rate of $s$ is exactly the same as that of the high frequency (HF) component, while when $a f^{2}>1$ the extrema rate of $s$ is exactly the same as that of the low frequency (LF) component. This proposition can easily be generalized to higher order derivatives. Indeed, let us assume that $s^{(2 k)}$ admits an extremum at $t_{0}$ :

$$
s^{(2 k+1)}\left(t_{0}\right) \propto \sin \left(2 \pi t_{0}\right)+a f^{2 k+1} \sin \left(2 \pi f t_{0}\right)=0,
$$

the derivative of order $2 k+2$ reads:

$$
s^{(2 k+2)}(t) \propto \cos (2 \pi t)+a f^{2 k+2} \cos (2 \pi f t)
$$

Then, we may write

$$
\begin{gathered}
\left|a f^{2 k+2} \cos \left(2 \pi f t_{0}\right)\right|<\left|\cos \left(2 \pi t_{0}\right)\right| \text { if } a f^{2 k+1}<1 \\
\left|a f^{2 k+2} \cos \left(2 \pi f t_{0}\right)\right|>|\cos (2 \pi t 0)| \text { if } a f^{2 k+2}>1
\end{gathered}
$$

the proof being the same as Proposition 1 of [15]. To summarize, the number of extrema of $s^{(2 k+1)}$ equals the number of extrema of the HF (resp. LF) component when $a f^{2 k+1}<1$ (resp. $a f^{2 k+2}>1$ ) because the sign of $s^{(2 k+2)}$ at $t_{0}$ is the same as that of the second derivative of the HF (resp. LF) component.

The separation of the $(a, f)$-plane by the curves $a f^{2 k+1}=1$ is illustrated on Figure 2 , for different derivation orders. We plot the difference between the number of extrema of $s^{(2 k)}$ and of the HF component $s_{1}$, for different orders $k$.

It is clear that the derivation decreases the contribution of the low frequency part in the signal, therefore a better extrema estimation is obtained by using higher derivation order. However, we are aware that in doing so, the noise is magnified. This leads us to build a trade-off procedure that determines a good derivation order. This procedure consists in computing the smallest $k$ such that $s^{(2 k)}$ has as many extrema as the HF mode and is as follows.

Let us denote by $t$ (resp. $\left.t^{(2)}, t^{(4)}\right)$ the locations of the extrema of $s$ (resp. $\left.s^{(2)}, s^{(4)}\right)$ and by $|X|$ the cardinal of the set $X$. The procedure to compute the $\hat{\theta}_{i}$, estimates of the extrema of the HF mode, is the following: 
- If $|t|=\left|t^{(2)}\right|$, then $\hat{\theta}=t$.

- Otherwise, if $\left|t^{(2)}\right|=\left|t^{(4)}\right|$ and $\left|t^{(2)}\right|>|t|$, then $\hat{\theta}=t^{(2)}$.

- Otherwise, $\hat{\theta}=t^{(4)}$.

To compute the second order derivative, we use the following fourth order formula:

$$
s^{\prime \prime}(t) \approx \frac{-s(t-2 \delta)+16 s(t-\delta)-30 s(t)+16 s(t+\delta)-s(t+2 \delta)}{12 \delta^{2}}
$$

where $\delta$ is the sampling period. The fourth order derivative is obtained by iterating this formula. On the boundaries, we use a shifted version of (12). Let us remark that $\delta$ must neither be too large, to avoid discretisation errors, nor too small, to avoid numerical errors caused by the roundoff unit. Note also that, to remove from our study potential sampling artifacts, we take the sampling rate at least equal to five times the Nyquist rate [16].

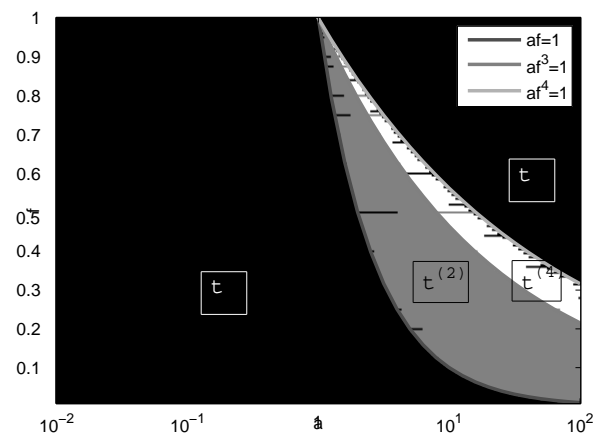

Fig. 3. Result of the selection method for the derivative order. From left to right, we display the regions where either $t$ (in black), $t^{(2)}$ (in gray) or $t^{(4)}$ (in white) are chosen.

The results of this procedure of automatic selection of the order of derivation are displayed on Figure 3 for the signal defined in (11). Each region corresponds to a particular value for $\hat{\theta}$ (i.e. $\hat{\theta} \in\left\{t, t^{(2)}, t^{(4)}\right\}$ ). Comparing this figure with previous graphics, we can conclude that this process manages to find the right number of extrema until $a f^{4}<1$. Then, if $a f^{4}>1$, we note that $\hat{\theta}=t$, which means that the HF mode cannot be separated from the rest of the signal. We could have expected the method to separate the HF mode from the rest of the signal until $a f^{5}=1$ which could be done using $t^{(4)}$. However, in the domain $a f^{5}<1<a f^{4}, t=t^{(2)}$ and corresponds to the number of extrema of the LF component, so that $t$ is chosen. 
Finally, to build the mean envelope, the classical EMD approach uses cubic splines. In our case, such a choice (i.e. $k=4$ ) would only be relevant for the extraction of the first IMF, but for complete decomposition, it is necessary to increase the order of the interpolation spline. Indeed, as the second IMF is extracted from $m$, if it were a cubic spline, the estimation of the extrema cannot be done using high order derivatives anymore. A possible solution would be to increase the order of the splines in the method, taking for example $k=6$.

\section{RESULTS AND EXPERIMENTS}

In the following tests, we will call our method "OS" for Optimization on Splines. The method "EMD" is the standard EMD computed with the code defined in [12]. In order to highlight the importance of the choice for $m_{0}$ on the different algorithms we also compute a modified version of the EMD, called "EMD-NI". The EMD-NI method is the standard EMD applied to $h_{0}=s-m_{0}$, where $m_{0}$ is computed using the approximation introduced at the begining of section III, with the improved estimation of the extrema of the HF mode.

\section{A. Mode Mixing}

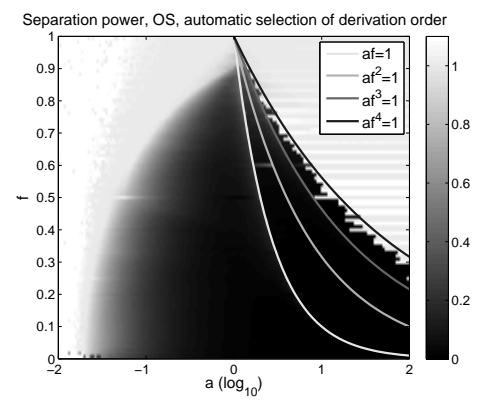

(A)

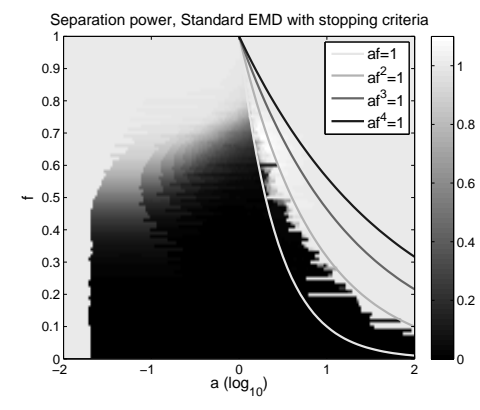

(B)

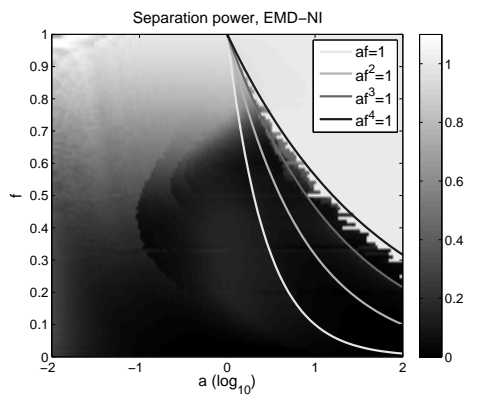

(C)

Fig. 4. (A): Computation of the mode separation with the proposed optimization algorithm $(\alpha=0.02)$, (B): Computation of the mode separation with the classical EMD algorithm, (C): Computation of the EMD on the signal $s-m_{0}$ where $m_{0}$ is computed using as in the optimization procedure.

We now illustrate the improvement brought about by using adaptively the order of derivative to build $m_{0}$ in terms of mode separation, when the signal $s$ is defined by (11). We shall then deduce that using higher order derivatives enables to better separate the HF component from the rest of the signal with an EMD-type algorithm. To measure this, we compute the cross-correlation between the obtained IMF and 
the desired mode as follows

$$
C_{1}=\frac{\left\|s_{1}-h\right\|}{\left\|s_{2}\right\|},
$$

where $s_{1}=\cos (2 \pi t)$ and $s_{2}=a \cos (2 \pi f t)$, which was already used in [15]. In the EMD-type method, the stopping criterion is that introduced in [12] which is based on two thresholds $\alpha$ and $\beta$ aimed at guaranteeing globally small fluctuations in the mean while taking into account locally large excursions. Considering the function $\sigma$ defined in (7), this amounts to iterate sifting until $\sigma(h)(t)<\alpha$ for some prescribed fraction $1-\gamma$ of the total duration, while $\sigma(h)(t)<\beta$ for the remaining fraction. One typically sets $\alpha \approx 0.05, \beta=10 \alpha$ and $\gamma=0.05$. For the sake of fair comparison, the value of the parameters $\alpha$ in the optimization problem (8) and in the standard EMD is set to 0.02. The result of the optimization procedure is shown on Figure 4 (A), while the separation results are shown for the original EMD on Figure 4 (B). To take into account, in the original EMD algorithm, that higher order derivatives may enable better HF component separation from the rest of the signal when the number of extrema of $s$ is lower than that of $s_{1}$, we compute the original EMD changing the first estimation of the mean envelope of $s$ by the cubic spline interpolant at $\left(\bar{t}_{i}, \bar{s}_{i}\right)$ where $\bar{t}_{i}$ is computed in the same way as in the initialization of the optimization procedure. This new version of the EMD is called EMD-NI, and the results are depicted on Figure $4(\mathrm{C})$.

These numerical results confirm the theoretical expectation: the separation of the mode depends on the order $2 k$ of the derivative used to compute the initial mean envelope, and the region where HF and LF components are not well separated indeed corresponds to $a f^{2 k+1}<1$ and to $a f^{2 k+2}>1$. To consider higher order derivatives significantly improves the modes separation and we note that our method behaves similarly to the improved EMD algorithm in terms of separation (Figure 4 (B) and (C)).

\section{B. Narrow-Band Signal Separation}

In this section, we evaluate different methods on narrow-band signal separation, that is we consider:

$$
s(t)=\sum_{i=1} s_{i}(t)
$$

where $s_{i}$ is an AM/FM sinusoidal component. We assume that the components are well separated in the frequency domain: for each $t$, all $s_{i}$ have distinct frequencies. Moreover, we suppose that each component satisfies the Bedrosian identity, so that we can write

$$
s_{i}(t)=a_{i}(t) \sin \left(\phi_{i}(t)+\phi_{0}\right)
$$



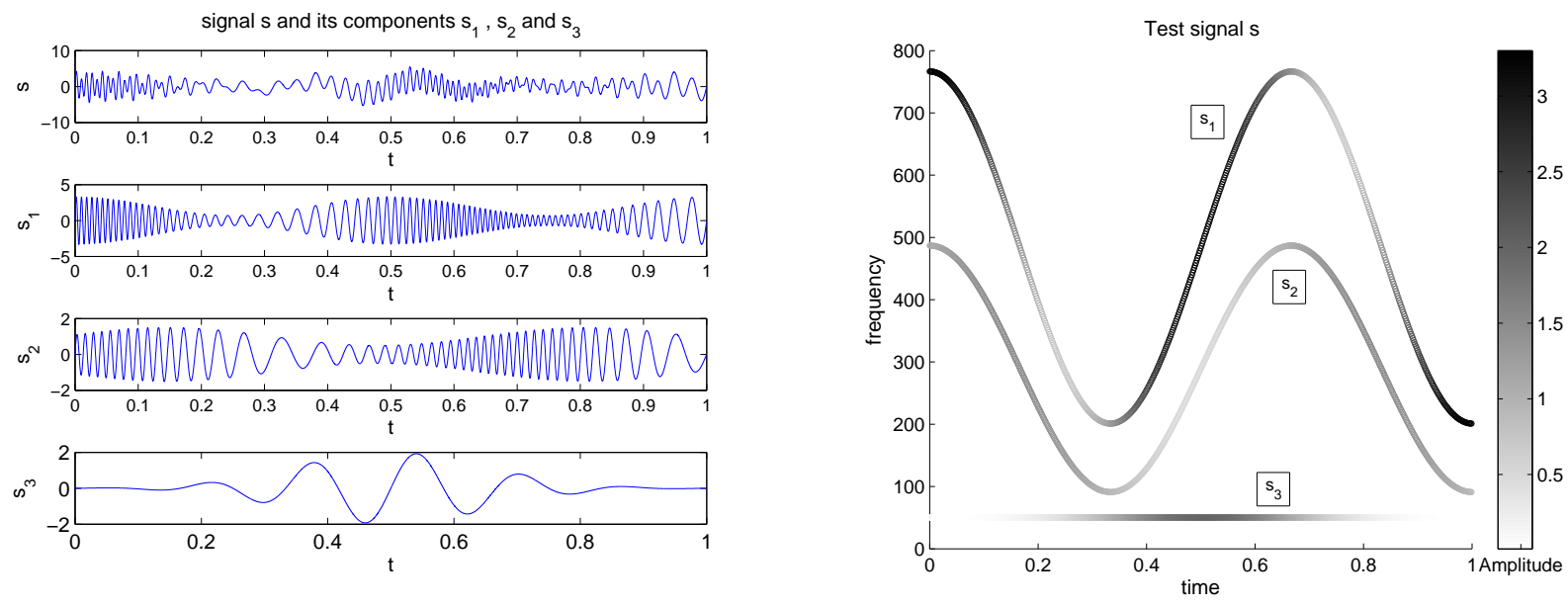

(A)

(B)

Fig. 5. (A): the three components $s_{1}, s_{2}, s_{3}$ of the test signal $s$, (B): the corresponding frequency representation.

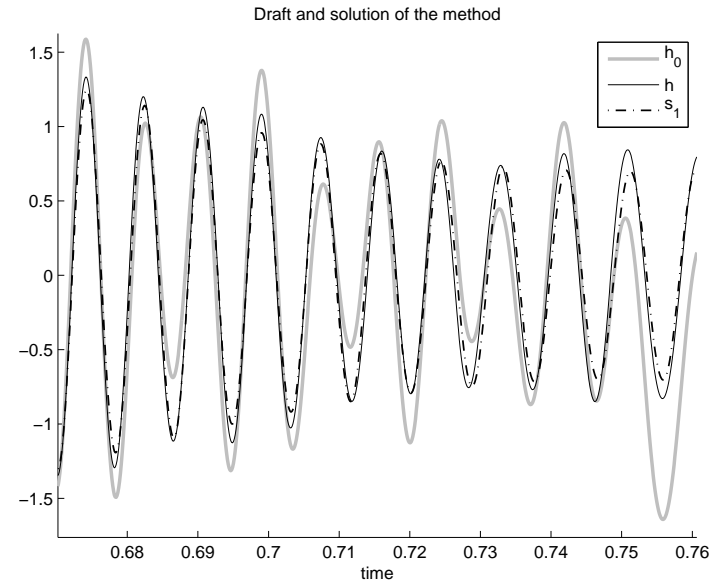

(A)

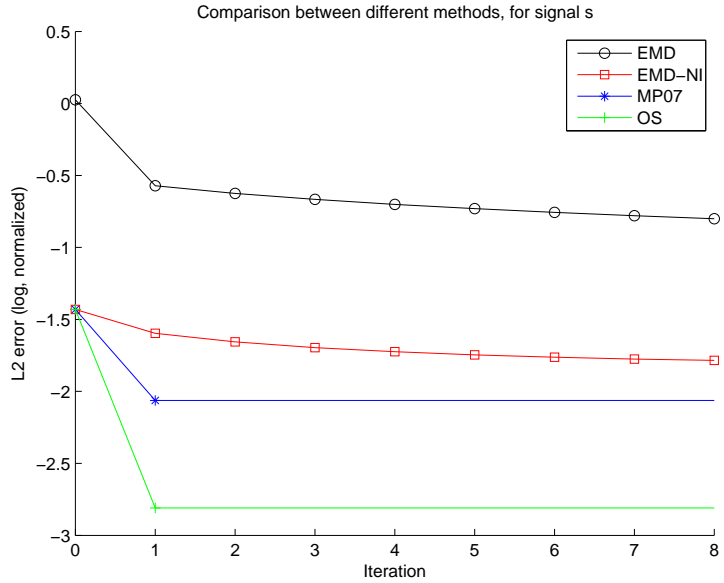

(B)

Fig. 6. (A): Result of the method: we display the draft $h_{0}$, the solution $h$ of the optimization procedure and the HF mode $s_{1}$ for a fraction of $s$. (B): Computation of (14) when the first mode is computed from the signal of Figure 5 using different methods.

where $a_{i}$ and $\phi_{i}$ are the instantaneous amplitude and phase. A typical example signal is shown on Figure 5 (A) along with its frequency representation. For such a signal, due to the absence of frequency interference the signals $s_{i}$ can be considered as uniquely defined and the different methods should separate them well. In this regard, we compare our new optimization approach to EMD-like algorithms. We compare 
the convergence to $s_{1}$ of the above introduced different methods computing:

$$
\log _{10}\left(\frac{\left\|s_{1}-h_{n}\right\|^{2}}{\left\|s_{1}\right\|^{2}}\right)
$$

$h_{n}$ being the pseudo-mode obtained after $n$ sitfing iterations in EMD-like algorithms (EMD and EMDNI). Of course, $n=1$ for the optimization process we propose, since the procedure is not iterative. The results of Figure 6 (B) shows the convergence of the original EMD to some $h$, which is definitely not $s_{1}$. To make a fair comparison with our method and since it is well known that, when the number of extrema is not correct at the first iteration, the sifting process may not converge, we also computed the EMD starting from $h_{0}=s-m_{0}, m_{0}$ being computed as at the end of section III. We again notice that the EMD converges towards a mode which is not $s_{1}$. On the contrary, our new optimization method OS computes a first mode $h_{1}$ closer to $s_{1}$.

\section{Variations on the Set of Constraints in the Optimization Method}

In this section, we compare the set of constraints used in our optimization procedure to that is used in [7]. Contrary to what is done in the current paper, the constraints in [7] where both of equality and inequality types. For the sake of consistency, we briefly recall how these constraints where designed for any signal $s$ with extrema located at $t_{i}$ 's. We consider the signal $h_{0}=s-m_{0}$, where $m_{0}$ is defined as previously, and where $\left(t_{i}\right)$ are this time the extrema of $h_{0}$. The idea developed in [7] is then to compute the first mode $h$ from $h_{0}$. In what follows, we denote by $m_{h}$ the sought mean envelope of $h$.

Assume that $h_{0}\left(t_{i}\right)$ is a minimum for $h_{0}$ and that $h_{0}\left(t_{i}\right)$ is an extremum for the sequence $\left(h_{0}\left(t_{i-2}\right), h_{0}\left(t_{i}\right)\right.$, $\left.h_{0}\left(t_{i+2}\right)\right)$. When $h_{0}\left(t_{i}\right)$ is an extremum for the latter sequence, the shape of the lower linear envelope is used to derive that of the upper. Indeed, let $\tilde{t}_{i}$ be the abscissa of the intersection (when it exists) of the straight lines $L_{1}: f_{1}(t)=\frac{h_{0}\left(t_{i}\right)-h_{0}\left(t_{i-2}\right)}{t_{i}-t_{i-2}} t+\frac{h_{0}\left(t_{i-1}\right)\left(t_{i}-t_{i-2}\right)-\left(h_{0}\left(t_{i}\right)-h_{0}\left(t_{i-2}\right)\right) t_{i-1}}{t_{i}-t_{i-2}}$ and $L_{2}: f_{2}(t)=$ $\frac{h_{0}\left(t_{i+2}\right)-h_{0}\left(t_{i}\right)}{t_{i+2}-t_{i}} t+\frac{h_{0}\left(t_{i+1}\right)\left(t_{i+2}-t_{i}\right)-\left(h_{0}\left(t_{i+2}\right)-h_{0}\left(t_{i}\right)\right) t_{i+1}}{t_{i+2}-t_{i}}$. If $\tilde{t}_{i}>t_{i}$, one imposes $(1 / 2)\left(f_{1}\left(t_{i}\right)+h_{0}\left(t_{i}\right)\right)=$ $m_{h}\left(t_{i}\right)$. Otherwise, one sets $(1 / 2)\left(f_{2}\left(t_{i}\right)+h_{0}\left(t_{i}\right)\right)=m_{h}\left(t_{i}\right)$. The same kind of computation can be carried out when $h_{0}\left(t_{i}\right)$ is an maximum for $h_{0}$ inverting the role of the upper and lower envelopes. This leads to a certain set of equality constraints.

Now, when the sequence $\left(h_{0}\left(t_{i-2}\right), h_{0}\left(t_{i}\right), h_{0}\left(t_{i+2}\right)\right)$ is monotonic, it gives rise to inequality constraints. Assume that $h_{0}$ has a minimum at $t_{i}$ (the following reasoning also holds when $h_{0}$ has a maximum at $t_{i}$ ). The symmetrical point $P_{i}$ of $\left(t_{i}, h_{0}\left(t_{i}\right)\right)$ with respect to $\left(t_{i}, m_{h}\left(t_{i}\right)\right)$ is $\left(t_{i}, 2 m_{h}\left(t_{i}\right)-h_{0}\left(t_{i}\right)\right)$. Following the classical EMD formulation, $P_{i}$ should therefore belong to the upper envelope. As $\left(t_{i-1}, h_{0}\left(t_{i-1}\right)\right)$ 
and $\left(t_{i+1}, h_{0}\left(t_{i+1}\right)\right)$ belong to the upper envelope, to preserve the monotonicity of the data, we impose:

$$
\begin{gathered}
\frac{\min \left(h_{0}\left(t_{i-1}\right), h_{0}\left(t_{i+1}\right)\right)+h_{0}\left(t_{i}\right)}{2} \leq m_{h}\left(t_{i}\right) \\
m_{h}\left(t_{i}\right) \leq \frac{\max \left(h_{0}\left(t_{i-1}\right), h_{0}\left(t_{i+1}\right)\right)+h_{0}\left(t_{i}\right)}{2} .
\end{gathered}
$$

These conditions, together with the cases where $h_{0}\left(t_{i}\right)$ is a maximum, leads to a set of inequality constraints. To compare this approach to that we have just developed, we replace the constraints $C$ in (8) by the above set of constraints, and we assume that $m_{h}$ is a piecewise cubic polynomial on the subdvision defined by the $t_{i}$, then it is entirely equivalent to the data $\Lambda=\left(m_{h}\left(t_{i}\right), m_{h}^{\prime}\left(t_{i}\right)\right)_{i=1, \cdots, L}$. The functional used to compute $m_{h}$ was then $\tilde{J}(m)=\left\|m^{\prime}\right\|^{2}$. Minimizing $\tilde{J}$ subject to above mentioned equality and inequality constraints, we obtain $\hat{m}_{h}$ and then define $h:=h_{0}-\hat{m}_{h}$. We display the result of this procedure, called "MP07", on Figure 6 (B), where we see that the obtained mode $h$ does not estimate $s_{1}$ as accurately as the newly proposed method.

\section{Entire decomposition and quasi-orthogonality}
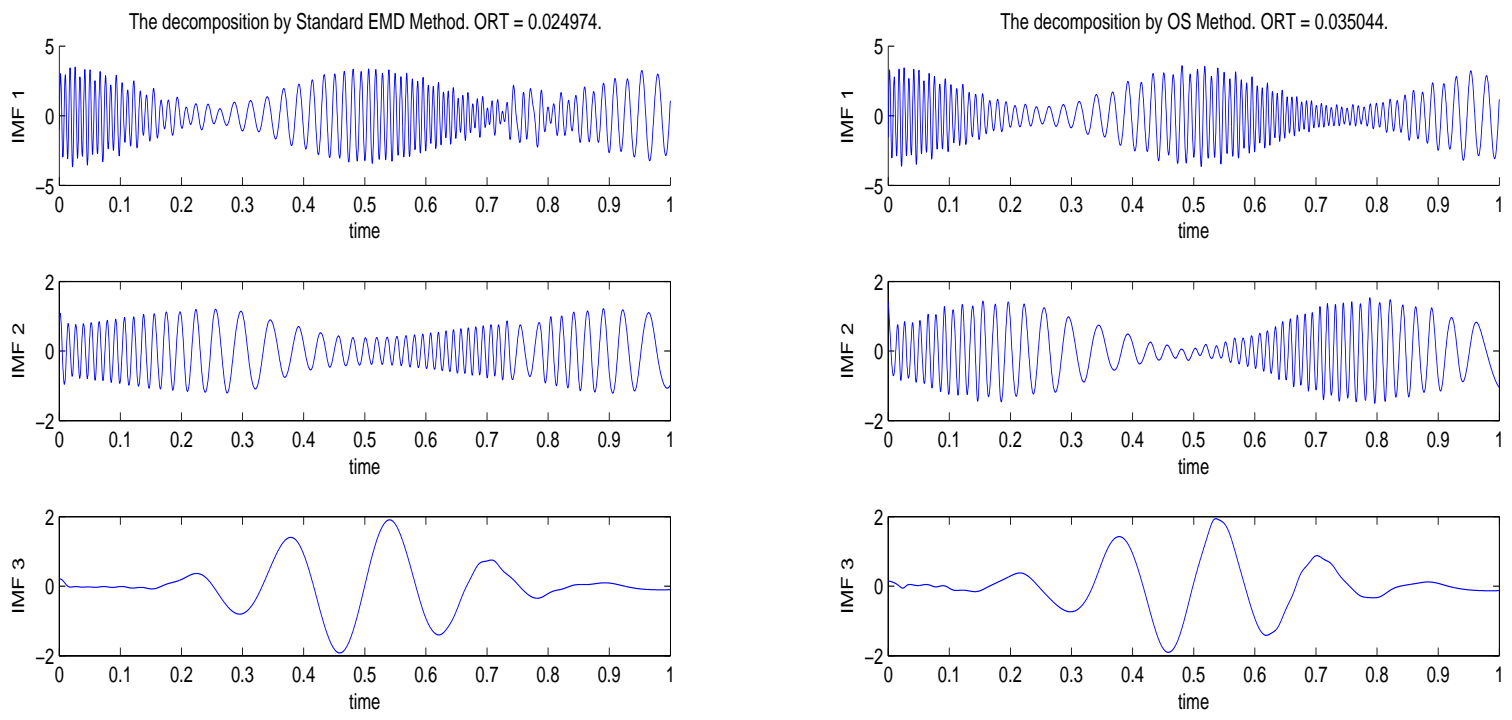

(A)

(B)

Fig. 7. (A): Result of the decomposition of the signal $s$ of Figure 5 using the standard EMD. (B): result of the OS method on the same signal.

Another major criterion to appreciate the quality of the decomposition is the orthogonality index, 
defined by

$$
i o=\frac{1}{\|s\|^{2}} \sum_{1 \leq i<j \leq N}\left\langle h_{i}, h_{j}\right\rangle,
$$

where $s$ is the signal and $\left(h_{i}\right)_{i}$ its IMF's.

On Figure 7, the entire decomposition of the signal defined on Figure 5 is displayed using either the original EMD or the new version we propose. The results exhibit very similar orthogonality index: 0.025 for the standard EMD and 0.035 for our method.

\section{CONCLUSION}

In this paper, we have introduced a new method to compute the intrinsic mode functions in the empirical mode decomposition based on an optimization procedure. Doing so, we avoid the classical problem posed by the convergence of the so-called sifting process that is the basis to the original EMD. We have shown that the proposed decomposition is adaptive, separates the modes better than the original EMD and leads to similar orthogonality indices. An extension to the bidimensional case looks, at first sight, relatively straightforward: indeed the constraints extend readily to interpolating functions on Delaunay triangulations, as used in [17]. This should be therefore the subject for further development.

\section{REFERENCES}

[1] N. Huang, Z. Shen, S. Long, M. Wu, H. Shih, Q. Zheng, N. Yen, C. Tung, and H. Liu, "The empirical mode decomposition and the Hilbert spectrum for nonlinear and non-stationary time series analysis," Proceedings of the Royal Society : Mathematical, Physical and Engineering Sciences, vol. 454, no. 1971, pp. 903-995, 1998.

[2] P. Flandrin, P. Gonçalves, and G. Rilling, "Detrending and denoising with empirical mode decompositions," in Proceedings of Eusipco, Wien (Austria), 2004, pp. 1581-1584.

[3] K. Coughlin and K. Tung, "11-year solar cycle in the stratosphere extracted by the empirical mode decomposition method," Advances in space research, vol. 34, no. 2, pp. 323-329, 2004.

[4] E. Delechelle, J. Lemoine, and O. Niang, "Empirical mode decomposition: an analytical approach for sifting process," IEEE Signal Process. Lett., vol. 12, no. 11, pp. 764-767, 2005.

[5] S. El Hadji, R. Alexandre, and A. Boudraa, "Analysis of Intrinsic Mode Functions: A PDE Approach,” Signal Processing Letters, IEEE, vol. 17, no. 4, pp. 398-401, 2010.

[6] S. Peng and W. Hwang, "Adaptive signal decomposition based on local narrow band signals," IEEE Trans. Signal Process., vol. 56, no. 7 Part 1, pp. 2669-2676, 2008.

[7] S. Meignen and V. Perrier, "A new formulation for empirical mode decomposition based on constrained optimization," IEEE Signal Process. Lett., vol. 14, no. 12, pp. 932-935, 2007.

[8] H. Hong, X. Wang, and Z. Tao, “Local Integral Mean-Based Sifting for Empirical Mode Decomposition,” IEEE Signal Process. Lett., vol. 16, no. 10, p. 841, 2009.

[9] Y. Kopsinis and S. McLaughlin, "Investigation and performance enhancement of the empirical mode decomposition method based on a heuristic search optimization approach,” IEEE Trans. Signal Process., vol. 56, no. 1, pp. 1-13, 2007. 
[10] E. Bedrosian, “A product theorem for Hilbert transforms," Proc. IEEE, no. 51, pp. 868-869, 1962.

[11] R. Sharpley and V. Vatchev, "Analysis of the intrinsic mode functions," Constructive Approximation, vol. 24, no. 1, pp. $17-47,2006$.

[12] G. Rilling, P. Flandrin, and P. Gonçalvès, "On empirical mode decomposition and its algorithms," in IEEE-EURASIP workshop on nonlinear signal and image processing NSIP-03, Grado (I), 2003.

[13] P. Flandrin, G. Rilling, and P. Goncalves, "Empirical mode decomposition as a filter bank," IEEE Signal Process. Lett., vol. 11, no. 2, pp. 112-114, 2004.

[14] C. De Boor, A practical guide to splines. Springer-Verlag, New York, 1978.

[15] G. Rilling and P. Flandrin, "One or two frequencies? The empirical mode decomposition answers," IEEE Trans. Signal Process., vol. 56, no. 1, pp. 85-95, 2008.

[16] N. Stevenson, M. Mesbah, and B. Boashash, "A sampling limit for the empirical mode decomposition," in The Eighth International Symposium on Signal Processing and Its Applications (ISSPA 2005), vol. 2. IEEE, 2010, pp. 647-650.

[17] C. Damerval, S. Meignen, and V. Perrier, "A fast algorithm for bidimensional EMD," Signal Processing Letters, IEEE, vol. 12, no. 10, pp. 701-704, 2005. 